\title{
Time for what? Breaking down temporal anticipation
}

\author{
Nir Shalev ${ }^{1,2}$, Anna C. Nobre ${ }^{1,2}$, Freek van Ede ${ }^{1 *}$ \\ ${ }^{1}$ Oxford Centre for Human Brain Activity, Wellcome Centre for Integrative Neuroimaging, Department of Psychiatry, \\ University of Oxford, UK \\ ${ }^{2}$ Department of Experimental Psychology, University of Oxford, UK
}

\begin{abstract}
* Corresponding author | Dr. Freek van Ede, Oxford Centre for Human Brain Activity, Wellcome Centre for Integrative Neuroimaging, Department of Psychiatry, University of Oxford, Warneford Hospital, Oxford OX3 7JX, UK. Email: frederik.vanede@,ohba.ox.ac.uk
\end{abstract}

Keywords: Temporal processing; Attention; Orienting; Perception; Action; Double dissociation; Basal ganglia; Cerebellum; Parkinson's disease; Cerebellar degeneration; Regularity; Rhythm; Association; Cognition; Performance; Interval judgement

\begin{abstract}
A variety of environmental regularities enable us to anticipate the timing of upcoming sensations and actions. A recent article reports a striking neural double dissociation between two distinct varieties of temporal anticipation. Looking forward, we consider further dissociations according to the goals for which temporal anticipation is used.
\end{abstract}

\section{Main text}

Temporal anticipation - the ability to predict the timing of forthcoming sensations and actions - is essential for adaptive behavior. Temporal anticipation can be formed based on multiple sources [1], such as temporal regularities that are currently present in the environment (e.g., a musical beat), temporal associations that we remember from prior experience (e.g., when a traffic light is likely to change), changes in the likelihood of an event with the passage of time ('hazard rates', e.g., waiting for an elevator to arrive), or knowledge of one's current position within a complex sequence of events (e.g., timing one's next dance move in a choreography). A central question in the study of temporal anticipation is whether a single set of brain areas is responsible for the alignment of the cognitive system to anticipated moments, or whether, in fact, there are multiple 'types' of temporal anticipation mediated by distinct brain systems.

In a recent $P N A S$ paper, Breska and Ivry [2] present compelling neuropsychological evidence for a double dissociation between rhythmic regularities vs. remembered temporal associations. Two groups of patients, one with Parkinson's Disease (PD; which involves a degeneration of the nigrostriatal system of the basal ganglia) and the other with Cerebellar Degeneration (CD), performed a task that required a speeded 
response to the identification of a perceptual target: a green colored shape, which appeared in $75 \%$ of the trials. Before the target appeared, participants viewed a sequence of centrally presented red shapes, followed by a gray shape which served as a warning signal for the imminent green-colored target. The experimental design consisted of three conditions: a random condition, in which the onset of targets, non-targets, and warning signals were temporally unpredictable; and two conditions in which the temporal onset of the targets was predictable. The two predictable conditions differed in how temporal anticipation was formed: In association-based predictions, prior exposure to specific intervals formed an associative memory template to guide temporal anticipation; In rhythm-based predictions, the stimulus stream followed a constant interval. The authors showed that individuals diagnosed with $\mathrm{CD}$ were selectively impaired in using association-based predictions (i.e. benefitting from such predictions in the experimental task), whereas individuals diagnosed with PD were selectively impaired in using rhythm-based predictions.

Therefore, although temporal anticipation may appear as a single, unified concept, the paper elegantly demonstrates that these two particular types of temporal regularities (temporal associations and rhythms) are dependent upon distinct brain areas (the cerebellum and the basal ganglia, respectively).

Of note, the article by Breska and Ivry did not aim to examine how time is kept by the brain, but rather how temporal predictability is used by the brain to guide ensuing behavior. To appreciate the implications of the work by Breska and Ivry, it is therefore essential to consider the specific way in which time was used in their task: to facilitate a speeded response, which may rely predominantly on response anticipation. Indeed, while their task is cast as a "visual detection task" to measure temporal attention, the task has in fact minimal perceptual demands, and group differences are only reported for response times. In contrast, direct effects on the perceptual sensitivity or accuracy for making visual discriminations are not reported. Also, the use of a go/no-go task design with a high proportion of targets promotes response anticipation further. Accordingly, the strength of the study is primarily in showing how different sources of temporal regularity can benefit behavioral responses, with little evidence for perceptual benefits per se.

The study therefore leaves open the possibility that whereas cerebellum and basal ganglia are causally and selectively involved when using association- and rhythm-based temporal regularities for anticipating action, a different set of brain areas may mediate temporal anticipation for perception (and yet other areas might mediate the awareness of time's passing). In other words, the goal of anticipation may provide another key 'axis' for dissociating brain systems that leverage temporal regularities to benefit performance. This raises the possibility of a 'double-double' dissociation among the potential sources of anticipation (e.g., rhythms or association, and possibly also hazard rates, sequences, ...) and which aspects of behavior are facilitated (e.g., action or perception, and possibly also working memory, learning, decision making, etc.).

One question in this regard, is whether timing for action and perception can be fully distinguished. Indeed, some have proposed that temporal anticipation for perception has motor origins [3]. Still, experimentally, there are ways to emphasize perceptual processing, while minimizing the contributions of motor anticipation. This includes introducing non-speeded tasks, or increasing perceptual demands and using a parametric approach to describe perceptual capacity (as in e.g. [4,5]). Future work could apply the 
experimental approach of Breska and Ivry to evaluate whether increasing the perceptual demands will reveal the same dissociation between cerebellar and basal ganglia patients, or whether possibly other areas are involved when temporal regularities are used for guiding attention exclusively to the perceptual features of the task.

The logic of our argument may be applicable at an even finer granularity, such as when considering distinct types of actions. For example, an interesting question is how to reconcile the marked inability of PD patients to benefit from rhythm-based prediction, as observed in the study by Breska and Ivry [2] with other literature demonstrating the utility of rhythm-based clinical interventions in PD, such as in facilitating gait [6]. Could it be that rhythm-based predictions improve natural walking behaviour but do not facilitate manual performance in the type of laboratory tasks employed by Breska and Ivry? This provides one example of how the use of the same type of temporal regularity (a rhythm) may be dissociated depending on the purpose it serves.

Though we emphasize the importance of investigating further dissociations, there is also room to investigate the extent to which shared neural circuits serve distinct 'timing functions'. For example, the cerebellum has also been implicated in explicit-duration judgments [7] and in perceptual anticipation [8]. With the growing emphasis on the temporal dimension of cognitive functions [1,9], we foresee that future research will identify circuits that participate in timing functions more generally as well as characterize specialized circuits sensitive to specific sources of temporal regularities and task demands.

With the logical strength of the double dissociation presented by Breska and Ivry, we refine our understanding of how temporal anticipation operates: it is mediated by different brain areas depending on the source of the 'temporal regularity' that affords temporal anticipation (i.e. the origin of temporal anticipation). Building on their work, we introduce the possibility of further distinctions according to the purpose (i.e. the goal) of temporal anticipation. This proposed line of future scientific inquiry can be expanded further: While the article by Breska and Ivry and the current Spotlight focus primarily on the use of time to guide anticipation and performance, time can also be the subject of perception in its own right (e.g., as in tasks requiring duration judgements). From the broader perspective of temporal cognition, one could consider time perception as another 'goal' that complements the goal of temporal anticipation. Indeed, those brain areas involved in 'using' temporal regularities to guide anticipation are not necessarily the same as those 'sensing' these regularities [10] - providing yet another interesting potential axis of dissociation.

\section{Acknowledgments}

This work was supported by a Wellcome Trust Senior Investigator Award (104571/Z/14/Z) and a James S. McDonnell Foundation Understanding Human Cognition Collaborative Award (220020448) to A.C.N, and by the NIHR Oxford Health Biomedical Research Centre. The Wellcome Centre for Integrative Neuroimaging is supported by core funding from the Wellcome Trust (203139/Z/16/Z). 


\section{References}

1 Nobre, A.C. and van Ede, F. (2017) Anticipated moments: temporal structure in attention. Nat. Rev. Neurosci. 19, 34-48

2 Breska, A. and Ivry, R.B. (2018) Double dissociation of single-interval and rhythmic temporal prediction in cerebellar degeneration and Parkinson's disease. Proc. Natl. Acad. Sci. 115, 1228312288

3 Morillon, B. and Baillet, S. (2017) Motor origin of temporal predictions in auditory attention. Proc. Natl. Acad. Sci. 114, E8913-E8921

$4 \quad$ Vangkilde, S. et al. (2013) Temporal expectancy in the context of a theory of visual attention. Philos. Trans. R. Soc. London. Series B, Biol. Sci. 368, 20130054

5 Rohenkohl, G. et al. (2012) Temporal expectation improves the quality of sensory information. J. Neurosci. 32, 8424-8428

6 Nombela, C. et al. (2013) Into the groove: Can rhythm influence Parkinson's disease? Neurosci. Biobehav. Rev. 37, 2564-2570

7 Grube, M. et al. (2010) Dissociation of duration-based and beat-based auditory timing in cerebellar degeneration. Proc. Natl. Acad. Sci. 107, 11597-11601

8 O'Reilly, J.X. et al. (2008) The cerebellum predicts the timing of perceptual events. J. Neurosci. 28, 2252-2260

9 Drayton, L. and Furman, M. (2018) Thy mind, thy brain and time. Trends Cogn. Sci. 41, 641-643

10 Coull, J.T. and Nobre, A.C. (2008) Dissociating explicit timing from temporal expectation with fMRI. Curr. Opin. Neurobiol. 18, 137-144 